\title{
El planeamiento estratégico y el trabajo en equipo docente de una institución educativa parroquial
}

\author{
Strategic planning and teaching teamwork in a parochial educational institution
}

Planejamento estratégico e ensino do trabalho em equipe em uma instituição de ensino paroquial

\section{ARTíCULO DE INVESTIGACIÓN}

\section{Zoila Josefina Vargas Holgado}

xiomara.voz@hotmail.com

https://orcid.org/0000-0001-9058-5089

Universidad Nacional Mayor de San Marcos, Lima-Perú

Carmen Vivar Bravo

yury1529@gmail.com

https://orcid.org/0000-0002-9871-5420

Universidad Micaela Bastidas, Abancay-Perú

\author{
Javier Vivar Bravo \\ xavibra7@gmail.com \\ https://orcid.org/0000-0003-2661-6447 \\ Universidad César Vallejo, Lima-Perú
}

Jessica Camayo Tovar

jesica.camayo@gmail.com

https://orcid.org/0000-0002-2206-860X

Universidad Nacional Mayor de San Marcos, Lima-Perú

Artículo recibido 10 de agosto 2021, arbitrado y aceptado 22 de septiembre 2021 y publicado 30 de diciembre 2021

RESUMEN

Desde la gestión directiva en las instituciones educativas se deben implementar acciones adecuada para el buen desempeño de los docentes, además, es importante diagnosticar las debilidades las cuales retrasan el cumplimiento de las metas, que lamentablemente repercutirán finalmenteenlos estudiantes, ental sentido una alternativa es la implementación de planes estratégico, desarrollados de manera colegiada fortaleciendo el trabajo cooperativo, por esta razón el objetivo de esta investigación fue determinar la relación entre el planeamiento estratégico y el trabajo en equipo docente de una institución educativa parroquial, se realizó un estudio de enfoque cuantitativo de tipo correlacional, empleando una población de 50 trabajadores, el procesamiento estadístico permitió concluir desde la percepción de los docentes que existe relación entre el planeamiento estratégico y el trabajo en equipo.

Palabras clave: Cooperación, Docentes, Gestión; Plan estratégico
ABSTRACT

RESUMO
From the directive management in the educational institutions, adequate actions must be implemented for the good performance of the teachers, in addition, it is important to diagnose the weaknesses which delay the fulfillment of the goals, which unfortunately will finally affect the students, in this sense an alternative is the implementation of strategic plans, developed in a collegiate manner, strengthening cooperative work, for this reason the objective of this research was to determine the relationship between strategic planning and teaching teamwork in a parochial educational institution, a focus study was carried out quantitative correlational type, using a population of 50 workers, statistical processing allowed to conclude from the teachers' perception that there is a relationship between strategic planning and teamwork.

Key words: Cooperation, teachers, management and strategic plan
A partir da gestão diretiva nas instituições de ensino, devem ser implementadas ações adequadas para o bom desempenho dos professores, além disso, é importante diagnosticar as fragilidades que atrasam o cumprimento dos objetivos, que infelizmente acabarão por afetar os alunos, neste sentido uma alternativa é a implantação de planos estratégicos, desenvolvidos de forma colegiada, fortalecendo o trabalho cooperativo, por isso o objetivo desta pesquisa foi verificar a relação entre o planejamento estratégico e o ensino do trabalho em equipe em uma instituição paroquial de ensino, foi realizado um estudo-foco No nosso tipo quantitativo correlacional, utilizando uma população de 50 trabalhadores, o tratamento estatístico permitiu concluir a partir da percepção dos professores que existe uma relação entre o planeamento estratégico e o trabalho em equipa.

Palavras-chave: Cooperação, professores, gestão e plano estratégico 


\section{INTRODUCCIÓN}

El año 2021 Perú y el mundo han sido afectados por la pandemia del COVID-19, esto significó que desde las instituciones educativas se impulsaran estrategias para adecuarse al nuevo sistema educativo, este contexto determinó que los directores asumieran su capacidad de liderazgo y organización, en tal sentido, el desarrollo de actitudes responsables que favorezcan al buen desempeño de su institución. El director como líder debe estar a la vanguardia tratando de evitar los modelos tradicionales empleados en la administración (Salvador et al., 2018), asimismo, no solo es crítico en su institución, tiene que iniciar y motivar cambios los cuales perduren en el tiempo (Anderson, 2010) liderazgo educativo demanda cualidades y características, promoviendo en su escuela una visión a futuro que contemple principios éticos y el desarrollo personal (Sierra Villamil, 2017).

Los problemas que afrontó la gestión directiva; no son exclusivos en el contexto de la emergencia sanitaria, lamentablemente hay dificultades que persisten en el tiempo, cómo es el trabajo en equipo entre los docentes, quizá el protagonismo personal o la debilidad de trabajar cooperativamente con otros han sido los limitantes, a los aspectos personales se sumó la sobrecarga laboral la cual se agudizó en el contexto de la emergencia sanitaria con el teletrabajo, debido a que no se encontraban preparados y les costó la adaptación, para GallardoGutiérrez (2020) los docentes durante la pandemia del COVID-19, comprendieron la necesidad de trabajar en equipo y tomar acciones de estrategias de apoyo para favorecer a los estudiantes.

\section{El planeamiento estratégico}

Apareció por los años cuarenta y su empleo se extendió por la década de los sesenta en lo que se convirtió en un instrumento importante para la organización (Ojeda, 2013) estuvo inclinada más al tema empresarial dentro de la administración, dándole un enfoque de gestión; sin embargo, en este estudio se aplicaron los principios de la planificación estratégica dentro de una institución educativa parroquial, en la cual el responsable de implementarla, socializarla y mantener su viabilidad fue el director haciendo uso de su liderazgo directivo.

Se consideró el planeamiento estratégico como un proceso que sirve para implementar y ejecutar, adecuadas acciones al interior de una organización, además son continuas y tienen como soporte la visión a futuro, para la toma de decisiones, en las cuales se debe considerar los riesgos y oportunidades el campo administrativo, la planeación estratégica es considerada una herramienta de gestión en la cual la meta es la eficacia y eficiencia. (Chiavenato y Sapiro, 2013; Armijo, 2011; Willardson, 2013). Para Herrera et al., (2018) quien citó a Rezende (2008) la planeación estratégica es activa dinámico y colaborativa, es decir involucra a todos los miembros de la institución. Por otro lado, Fernández Hurtado et al., (2019) sostuvieron que, en el planeamiento estratégico el principal propósito es elaborar una guía, la cual brinde orientaciones donde se fijen con claridad los objetivos que se propone la institución y sean factibles de ejecutar. Además, implementarla puede contribuir a conseguir la excelencia (Almuiñas y Galarza, 2019). En este estudio se consideró dos dimensiones dentro del planeamiento estratégico, el conocimiento y evaluación.

Con respecto a la primera dimensión que es el conocimiento, dentro de la institución educativa en Perú, los directivos y docentes deben estar informados de dos aspectos básicos; primero las normativas que emita el Ministerio de Educación (MINEDU), como son la Ley General de Educación, 
La Ley de la Carrera Pública Magisterial, además estar pendiente de las resoluciones que se brindan con frecuencia con respecto al servicio educativo, esto es fundamental porque al implementar un plan o proyecto es necesario estar al tanto hasta donde pueden llegar los alcances y cuáles son las limitaciones; el segundo aspecto es el conocimiento del currículo, el cual forma parte del desempeño pedagógico y esencial para la buena práctica profesional. También es indispensable, tomar en cuenta que toda institución educativa emite sus documentos de gestión, los cuales son elaborados de forma consensuada, mediante reuniones colegiada entre los miembros de la comunidad educativa, los cuales son un insumo más para elaborar el plan estratégico.

Con respecto a la primera dimensión conocimiento resultó necesario para la implementación del plan estratégico el análisis situacional de la institución, en las escuelas esto se realiza con el diagnóstico del FODA; Fortalezas, Oportunidades, Debilidades y Amenazas, lo que permite tener una visión panorámica de la situación real, la construcción de este documento involucra a todos los miembros de la comunidad educativa, de acuerdo a Beach (2015) en el análisis del planeamiento estratégico, contribuye para que los líderes piensen en el futuro, comprendiendo el presente, en esta línea Babaoglan (2015) sostuvo que, planificar en una institución educativa debe ser una prioridad para los directivos, en tal sentido que le permita tomar decisiones estratégicas.

La segunda dimensión de planeamiento estratégico es la evaluación, en la cual se comprueba su efectividad, de no evidenciarse se realizarán los ajustes respetivos para una nueva ejecución, es importante que en esta fase, los miembros de las escuelas, no tengan temor a ser evaluados, todos podemos ser factibles de errores y al igual que cuando se evalúa a los estudiantes se les brinda retroalimentación, es necesaria en una institución al implementar planes o proyectos realizar esta labor con el equipo en el cual se ejecutó.

\section{El trabajo en equipo}

Las instituciones educativas son un espacio de interacción que fortalece las relaciones sociales, en la cual se buscan practicas democrática, equitativas e inclusiva; que involucra a todos los actores de la comunidad educativa, por tanto la sumatoria de esfuerzos conlleva al trabajo en equipo, los estudiantes desde las aula trabajan de manera cooperativa se busca mantener una adecuadas convivencia con los padres, en tal sentido los docentes deben ser un ejemplo de integración y colaboración, evidenciar que ellos también pueden trabajar de manera organizada y solidaria, el desempeño que realicen tendrá como resultado un buen clima laboral que repercutirá en beneficio de sus estudiantes. Sin embargo, la realidad más de una vez ha demostrado que a los individuos les cuestas la integración, adaptarse a los estilos y costumbres de otros por eso se consideró en este estudio que una adecuada planificación estratégica, debe incluir al equipo de trabajadores.

Torelles et. al (2013) sostuvieron que, para trabajar en equipo se necesita contar con habilidades y aptitudes, para adaptarse pudiendo juntos conseguir resultados en un contexto determinado.

La investigación que se realizó en la institución educativa parroquial se debió a que se encontraron debilidades en el desempeño profesional de los 
docentes al cumplir funciones académicas y curriculares, esta dificultad preocupó, porque desde la gestión y de acorde al las recomendaciones del Ministerio de Educación (Minedu), se debe promover el trabajo colegiado, e incluso dentro de la calendarización del periodo lectivo en el Plan Anual de Trabajo se incluyen las jornadas de integración; no obstante estas exigencias, se observó deficiencias, en tal sentido la implementación de medidas como un plan estratégico impulsado por el director como líder escolar era imprescindible.

Tomando en cuenta lo expuesto, evidenció la importancia del director desde su gestión contribuya a generar planes o proyectos con los docentes, para mejorar la interacción entre ellos y brindar un servicio educativo de calidad, se formuló como objetivo en esta investigación determinar la relación el planeamiento estratégico y el trabajo en equipo docente en una institución educativa parroquial este estudio se consideró de impacto debido a que, se exploró la necesidad de desde la gestión en implementar como parte de la visión institucional acciones generadoras de cambios, acorde a los indicadores de calidad que es necesario en los planes de mejora continua y la planeación estratégica contribuye al buen funcionamiento de la institución educativa y finalmente favorezcan a los estudiantes.

\section{MÉTODO}

El método empleado en este estudio fue de enfoque cuantitativo, de tipo descriptivo correlacional, el estudio fue realizado en una institución educativa parroquial ubicada en Lima Metropolitana, se realizó a una población de 50 participantes, se tuvo como criterio de inclusión que todos fueran trabajadores activos.

Se diseñó un instrumento en escala de Likert con quince ítems para medir la percepción del planeamiento estratégico, al cual se le realizó la prueba de validación mediante juicio de expertos y de confiabilidad se utilizó el alfa de Cronbach. De acuerdo al procesamiento del instrumento que midió la variable planeamiento estratégico, mediante el coeficiente del Alfa de Cronbach, dio como resultado 0.834 , lo que permitió afirmar que el instrumento tuvo una fuerte confiabilidad para su aplicación.

\section{RESULTADOS}

En este estudio se consideró como hipótesis general, existe relación entre la planeación estratégica y el trabajo en equipo docente de una institución educativa parroquial, en tal sentido el procesamiento estadístico rechazó la hipótesis nula, a continuación, se detallan los resultados de acuerdo a las dimensiones que se consideró en la variable planeación estratégica: conocimiento y evaluación. 
Tabla 1. Coeficiente de correlación rho de Spearman de la planeación estratégica y el trabajo en equipo docente de una institución educativa parroquial.

\begin{tabular}{crlrr}
\hline & & Planeación estratégica & Trabajo en equipo \\
\hline Rho de & Planeación & Coeficiente de correlación & 1,000 &, $762^{* *}$ \\
Spearman & estratégica & Sig. (bilateral) &. &, 000 \\
& & $\mathrm{~N}$ & 50 & 50 \\
& & &, $762^{\star *}$ & 1,000 \\
& Trabajo & Coeficiente de correlación &, 000 & $\cdot$ \\
& en equipo & Sig. (bilateral) & 50 & 50 \\
\hline
\end{tabular}

**. La correlación es significativa en el nivel 0,01 (2 colas).

En la Tabla 1 se observó que el valor del coeficiente de correlación rho de Spearman es $\mathrm{r}=$ 0,762 el cual se interpreta como una correlación alta y positiva entre las variables planeación estratégica y el trabajo en equipo.

Tabla 2. Coeficiente de correlación rho de Spearman de la dimensión conocimiento y el trabajo en equipo.

\begin{tabular}{|c|c|c|c|c|}
\hline & & & Conocimiento & Trabajo en equipo \\
\hline \multirow{6}{*}{$\begin{array}{c}\text { Rho de } \\
\text { Spearman }\end{array}$} & \multirow[t]{3}{*}{ Conocimiento } & Coeficiente de correlación & 1,000 &, $641^{* *}$ \\
\hline & & Sig. (bilateral) & . & 000 \\
\hline & & $\mathrm{N}$ & 50 & 50 \\
\hline & \multirow{3}{*}{$\begin{array}{c}\text { Trabajo } \\
\text { en equipo }\end{array}$} & Coeficiente de correlación &, $641^{\star *}$ & 1,000 \\
\hline & & Sig. (bilateral) &, 000 & . \\
\hline & & $\mathrm{N}$ & 50 & 50 \\
\hline
\end{tabular}

**. La correlación es significativa en el nivel 0,01 (2 colas).

En la Tabla 2 se observó que el valor del coeficiente de correlación rho de Spearman es $\mathrm{r}=0,641$ el cual se interpreta como una correlación alta positiva entre la variable comunicación y el trabajo en equipo.

Tabla 3. Coeficiente de correlación rho de Spearman de la dimensión evaluación y el trabajo en equipo.

\begin{tabular}{|c|c|c|c|c|}
\hline & & & Evaluación & Trabajo en equipo \\
\hline \multirow{6}{*}{$\begin{array}{l}\text { Rho de } \\
\text { Spearman }\end{array}$} & \multirow[t]{3}{*}{ Evaluación } & Coeficiente de correlación & 1,000 &, $592^{\star *}$ \\
\hline & & Sig. (bilateral) & . & 000 \\
\hline & & $\mathrm{N}$ & 50 & 50 \\
\hline & \multirow{3}{*}{$\begin{array}{c}\text { Trabajo } \\
\text { en equipo }\end{array}$} & Coeficiente de correlación &, $592^{\star *}$ & 1,000 \\
\hline & & Sig. (bilateral) &, 000 & . \\
\hline & & $\mathrm{N}$ & 50 & 50 \\
\hline
\end{tabular}

**. La correlación es significativa en el nivel 0,01 (2 colas). 
En la Tabla 3 se observó que el valor del coeficiente de correlación rho de Spearman es $\mathrm{r}=0,592$ el cual se interpreta como una correlación moderada positiva entre las variables evaluación y el trabajo en equipo.

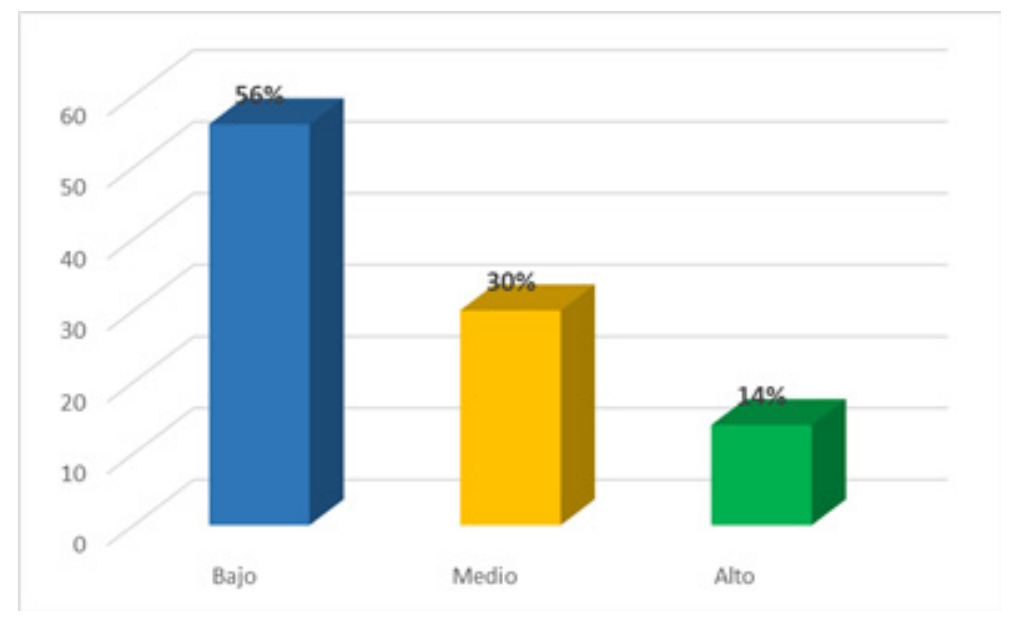

Figura 1. Diagrama de la distribución de frecuencia de la variable planeación estratégica según los docentes del Institución Educativa Parroquial Santísimo Salvador.

De acuerdo a los datos de la estadística descriptiva en la Figura 1; permitió inferir las siguientes apreciaciones, en primer lugar es evidente la percepción de los docentes se encuentra en un nivel bajo con un $56 \%$, en tal sentido un importante número de trabajadores de la institución parroquial donde se aplicó la investigación, son conscientes de las deficiencias del trabajo en equipo, frente a un $30 \%$ en el nivel medio y un $14 \%$ en el nivel alto, el cual sería productivo factible de estado también profundizar a estos últimos, porque ellos consideran la labor en la institución de manera satisfactoria.

\section{DISCUSIÓN}

El resultado obtenido en el procesamiento estadístico se comprobó que, existe relación entre el planeamiento estratégico y el trabajo en equipo, en esta línea de los beneficios de implementarla coincidió el estudio de Fernández Hurtado et al.,
(2019) quienes concluyeron que, no depende si la compañía es grande o pequeña; sin embargo una adecuada ejecución puede llevar al éxito y una mal plan al fracaso. Por otro lado, Ore, Olortegui y Ponce (2020) concuerdan en la definición al seguir los procesos, el riguroso cumplimiento de los objetivos ayudará a mejorar la eficacia y eficiencia en una constitución. Cervantes, Muñoz e Inda (2020) evidenciaron que cuando se dan buenos resultados del trabajo en equipo, el cual se demostró en su estudio en la calidad de servicio el cual puede ser percibido por los clientes, por tal razón en una institución educativa el buen desempeño de los docentes en forma colaborativa se reflejará en adecuado rendimiento académico; sin embargo Freire-Andrade et al., (2019) concluyeron que es necesario la sinergia entre el modelo que se emplee en el planeamiento estratégico y la gestión, reafirmando la propuesta inicial que se realizó en 
este estudio la importancia de los líderes para la ejecución de un plan.

Para Robles et al. (2017), quienes realizaron el estudio del desarrollo de planes estratégicos en profesionales de salud, determinaron que en la implementación, se debe considerar a los actores; sus objetivos e interés al interior de la instrucción; en la misma línea que se determinó en este estudio la necedad de involucrar a los docentes. Similar recomendación se encontraron en el estudio de Ojeda (2013) realizaron en México determinaron que, cada vez más instituciones educativas se suman a elaborar planes estratégicos, además son diseñados en forma cooperativa, por tal razón la consulta es necesaria.

EnelestudiodeOztürkyGenç(2021) sostuvieron que, en Turquía se impulsó la implementación de planes estratégicos en las universidades como parte de las políticas públicas; pero esto no fue una garantía para la mejora de la cultura organizacional, para su elaboración se consultaba a los miembros de las universidades; sin embargo existía ya una forma de organización en el clima institucional que los autores llamaron de clanes muy marcada, los investigadores evidenciaron con el procesamiento estadístico la percepción de las personas encuestadas de estas instituciones, la necesidad de instaurar un sistema adaptativo a los nuevos tiempos, e incluir nuevas alternativas; este estudio demostró que a pesar de implementarse por mucho tiempo planes estratégicos supervisados desde la gestión pública, no consiguen el ideal esperado si el grupo humano no colabora en las mejoras.

La investigación de (León, 2016) realizado en Costa Rica, exploró la implementación planes estratégicos y el desempeño profesional docente, cuyos resultados fueron muy similares a los que obtuvimos; porque demostró en sus resultados y conclusiones, la percepción de los docentes en qué adecuada participación en los planes contribuye a un mejor trabajo profesional; además señalaron la importancia que desde la gestión directiva se prioriza temas a incluir, coincidiendo en la trascendencia que tienen los líderes escolares cuando se decide impulsar un plan estratégico, asimismo la importante de la motivación ara que la ejecución de este permanezca vigente en el tiempo propuesto.

\section{CONCLUSIONES}

Se concluyó que existió relación entre el planeamiento estratégico y el trabajo en equipo docente; además, el principal responsable en promover los cambios en las instituciones educativas fue el director como líder, el cual haciendo uso de este atributo debe contar con la capacidad de poder convocar a sus docentes en la toma de decisiones, las cuales favorezcan a las mejoras de su escuela, asimismo, propiciar la integración de manera colaborativa que permita el trabajo en equipos. La evaluación de un plan estratégico se evidenció debe ser constante y el equipo recibir retroalimentación para cumplir las metas trazadas.

La implementación de planes estratégicos puede tener efectos positivos, aunque se observó de acuerdo a Oztürk y Genç (2021), que los resultados necesariamente no siempre son los esperados, debido a múltiples factores como: culturales, complicaciones para entender las personas y resistencia al cambio.

Finalmente, para la permanecía en el tiempo de los planes estratégicos fue importante la motivación permanente, impulsada por el director como líder siguiendo la ruta de la visión que elaboró la institución educativa. 


\section{REFERENCIAS}

Armijo, M. (2011). Planificación estratégica e indicadores de desempeño en el sector público. Chile, Santiago de Chile: CEPAL.

Anderson, S. (2010). Liderazgo directivo: Claves para una mejor escuela. Psicoperspectivas, 9(2), 34-52. https://doi.org/10.5027/ PSICOPERSPECTIVAS-VOL9-ISSUE2FULLTEXT-127

Almuiñas Rivero, J., \& Galarza López, J. (2019). Evaluation of Strategic Planning in Higher Education Institutions in Cuba. Methodology Used and Results Obtained. 16.

Babaoglan, E. (2015). Strategic planning in education in Turkey. Educational Planning, 22(2), 35-39.

Beach R., y R. L. (2015). A Discussion of Strategic Planning as Understood through the Theory of Planning and Its Relevance to Education. Educational Planning, 22(2), 5-16.

Cervantes, G., Muñoz, G., \& Inda, A. (2020). El trabajo en equipo y su efecto en la calidad del servicio a clientes. Revista Espacios, 41(14), 2737. http://ww.revistaespacios.com/a20v41n14/ a20v41n14p27.pdf

Chiavenato, I y Sapiro, A. (2011). Planeación estratégica. Fundamentos y aplicaciones. (2da ed.). México, México D.F.: McGraw-Hill/ Interamericana Editores, S.A. de C.V.

Fernández Hurtado, S. R., Martínez Martínez, L. Á., \& Ngono Fouda, R. A. (2019). Barreras que dificultan la planeación estratégica en las organizaciones. Tendencias, 20(1), 254-279. https://doi.org/10.22267/rtend.192001.108

Freire-Andrade, V., Juan, y Rocha-Hoyos, C. (2019). Análisis de la planificación estratégica para la gestión de las universidades particulares. Una alternativa exitosa Analysis of strategic planning for the management of private universities. A successful alternative. Rev. Espacios, 40(2), 1-7. https://www.revistaespacios.com/a19v40n02/ a19v40n02p25.pdf

Gallardo-Gutiérrez, A. (2020). Saberes docentes ante la pandemia Tensiones y alternativas. Perfiles Educativos, XLII, 32-38.
Herrera, J. I., Parrilla, Á., Blanco, A., y Guevara, G. (2018). Teacher Training for Inclusive Education. A Challenge from the National University of Education in Ecuador. Revista Latinoamericana de Educación Inclusiva, 12(1), 21-38. https:// www.scielo.cl/pdf/rlei/v12n1/0718-7378rlei-12-01-00021.pdf

León, H. (2016). Gestión de la planificación estratégica para el desarrollo profesional docente.pdf. Gesdtión de La Educaión, 6, 23-52. file://C:/Users/USUARIO/Desktop/ATE/Vista de Gestión de la planificación estratégica para el desarrollo profesional docente.pdf

Ore, H.; Olortegui, E. y Ponce, D. (2020). Planeamiento estratégico como instrumento de gestión en las empresas: Revisión bibliográfica. Revista Pakamuros, 8(4), pp. 31 - 44. Consultado en http://revistas.unj.edu.pe/index. php/pakamuros/article/view/147/127

Ojeda Ramírez, D. M. M. (2013). La planificación estratégica en las instituciones de educación superior mexicanas: De la retórica a la práctica. CPU-e, Revista de Investigación Educativa, 16, 110-118. https://doi.org/10.25009/cpue. v0i16.203

Oztürk, A. \& Genç, E. (2021) Preliminary identification of organizational culture styles in the process of strategic planning: employee perception in the turkish public higher educationñ. Journal of Economics and Administrative Sciences Faculty. Volume: 8 Issue: 1 p.231-255file://C:/Users/USUARIO/Desktop/ ATE/10.30798-makuiibf.799294-1306840.pdf

Robles, R., Serrano, H., Serrano, G., Gaibor, F., Armijo, G., y Fernandez, A. (2017). Retos de la planificación estratégica en instituciones de salud. Revista Cubana de Investigaciones Biomédicas., 36(3), 1-6.

Salvador Cornelio, E. M., y Sánchez Ortega, J. A. (2018). Liderazgo de los directivos y compromiso organizacional Docente. Revista de Investigaciones Altoandinas - Journal of High Andean Research, 20(1), 115-124. https:// doi.org/10.18271/ria.2018.335 
Sierra Villamil, G. M. (2017). Liderazgo educativo en el siglo XXI, desde la perspectiva del emprendimiento sostenible. Revista EAN, 81, 111.https://doi.org/10.21158/01208160. n81.2016.1562
Willardson, S. (2013). Strategic intelligence during coin detention operations relational data and understanding latent terror networks. Defense 